\title{
SCHIZOPHRENIA PATIENTS: PHYSICAL ACTIVITY IS AND REMAINS AN ORGANISMIC NECESSITY
}

Singapore Med J 2013; 54(4): 236-237 doi: 10.11622/smedj.2013081

I thank Said et al for their interesting article, "Metabolic syndrome and cardiovascular risk among patients with schizophrenia receiving antipsychotics in Malaysia",(1) published in a recent issue of this journal. The high prevalence of metabolic syndrome (MetS) in patients with schizophrenia, as noted by the authors, has been confirmed by another recent study in Singapore, which revealed a prevalence of $46.0 \% .^{(2)}$ The authors rightly pointed out that these patients are in urgent need of systematic screening, as well as pharmacological and non-pharmacological interventions. I wish to make an additional comment regarding regular physical activity (including aerobic and resistance exercise), which is essential for the prevention and treatment of MetS and its components. ${ }^{(3)}$

Firstly, it is to be assumed that among the schizophrenia patients in the study population, the current level of physical activity was lower in patients with MetS than in those without MetS. While Said et al had, in general, accurately indicated that sedentary habits can be associated with the disease, they unfortunately made no indication of the patients' current levels of physical activity. ${ }^{(1)}$ Recently, Vancampfort et al found that patients with schizophrenia are sedentary for two hours more per day than healthy controls (8.5 hours vs. 6.21 hours). More sedentary time was associated with increases in body mass index (BMI), waist circumference (WC), fasting glucose concentrations, as well as higher rates of MetS and negative cognitive symptoms. ${ }^{(4)}$ For example, standing up frequently is a simple but highly efficient daily exercise for schizophrenia patients, as it would not only increase blood circulation in the brain and strengthen the supportive muscular system as a whole, but also result in favourable changes in MetS components. Although it may be difficult to persuade these patients to stand up frequently, they should be encouraged to do so, as the benefits will become evident to the patients in the future.

Secondly, aerobic exercises, yoga and resistance training can help to reduce negative mental symptoms such as a lack of emotion, lethargy, disinterest, depression and anxiety in patients with schizophrenia. They also improve the quality of life and somatic health. ${ }^{(5)}$ Furthermore, physical activity has been known to induce adult hippocampal neurogenesis and plasticity through the formation of brain-derived neurotrophic factor (BDNF), vascular endothelial growth factor (VEGF), $\beta$-endorphin and serotonin. For example, a recent randomised controlled study demonstrated that a 3-month period of aerobic exercise training $(3 \times 30$ minutes of cycling per week) induced an increase of hippocampal volume by $12 \%$ and improved short-term memory (as assessed by Rey Auditory Verbal Learning Test) in chronic schizophrenia patients. ${ }^{(6)}$ In schizophrenia patients, participation in sports also seems to result in an activation of the body-selective extrastriate body area in the posterior temporal-occipital cortex, which is associated with an improvement in the general psychopathology scale of the Positive and Negative Syndrome Scale. ${ }^{(7)}$ This implies that physical activity directly contributes to brain health.

Thirdly, it would be desirable for clinicians to increase the use of the well-validated 6-Minute Walk Test (6MWT) as part of their multidisciplinary screening assessments and monitoring practices for schizophrenia patients. It has been demonstrated that the distance walked in the 6MWT is significantly associated with BMI, WC, sports participation, leisure-time physical activity, negative and depressive symptoms and the daily antipsychotic dose. ${ }^{(8,9)}$ In addition, schizophrenia patients with MetS have worse performance in the 6MWT than those with non-MetS $\left(527.6 \pm 108.9 \mathrm{~m}\right.$ vs. $610.0 \pm 93.7 \mathrm{~m}$, respectively). ${ }^{(9)}$

In conclusion, more consideration should be given to physical activities and exercises within the overall intervention concept of local district psychiatric healthcare systems. This approach should be taken from the start of schizophrenic disease. In my opinion, we should not forget the old, yet timeless rule, as expressed by the French physician Joseph Clément Tissot (1747-1826), "Physical activity can often be used to replace medicines; however, no medicine could ever possibly take the place of physical activity."(10) Accordingly, in lifestyle management, regular physical activity must not be conceived as a temporary intervention - a view widely held among patients with schizophrenia receiving antipsychotics - but must be increasingly integrated into daily routines.

\section{Yours sincerely,}

\section{Martin Hofmeister}

Consumer Centre of the German Federal State of Bavaria, Department of Food and Nutrition, Mozartstraße 9 , D-80336 Munich, Germany. hofmeister@vzbayern.de

\section{REFERENCES}

1. Said MA, Sulaiman AH, Habil MH, et al. Metabolic syndrome and cardiovascular risk among patients with schizophrenia receiving antipsychotics in Malaysia. Singapore Med J 2012; 53:801-7.

2. Lee J, Nurjono M, Wong A, Salim A. Prevalence of metabolic syndrome among patients with schizophrenia in Singapore. Ann Acad Med Singapore 2012; 41:457-62.

3. Ilanne-Parikka P, Laaksonen DE, Eriksson JG, et al. Leisure-time physical activity and the metabolic syndrome in the Finnish diabetes prevention study. Diabetes Care 2010; 33:1610-7.

4. Vancampfort D, Probst M, Knapen J, Carraro A, et al. Associations between sedentary behaviour and metabolic parameters in patients with schizophrenia. Psychiatry Res 2012; 200:73-8. 
5. Vancampfort D, Probst M, Helvik Skjaerven L, et al. Systematic review of the benefits of physical therapy within a multidisciplinary care approach for people with schizophrenia. Phys Ther 2012; 92:11-23.

6. Pajonk FG, Wobrock T, Gruber O, et al. Hippocampal plasticity in response to exercise in schizophrenia. Arch Gen Psychiatry 2010; 67:133-43.

7. Takahashi H, Sassa T, Shibuya T, et al. Effects of sports participation on psychiatric symptoms and brain activations during sports observation in schizophrenia. Transl Psychiatry 2012; 2:e96.

8. Vancampfort D, Sweers K, Probst M, et al. Association of the metabolic syndrome with physical activity performance in patients with schizophrenia. Diabetes Metab 2011; 37:318-23.

9. Vancampfort D, Probst M, Scheewe T, et al. The functional exercise capacity is correlated with global functioning in patients with schizophrenia. Acta Psychiatr Scand 2012; 125:382-7.

10. Tissot JC. [Medical and surgical gymnastics or test regarding the curative benefits of physical activity or various physical exercises and rest in the context of diseases]. Leipzig: Jacobäer, 1782. German. 